\title{
Editorial
}

\section{Proximal Prematurities in Prosthodontics}

The term 'Prematurity' in the 'Glossary of Prosthodontic terms' (GPT) directed towards the 'Occlusal deflective contacts' and is defined as a contact that displaces a tooth, diverts the mandible from its intended movement, or displaces a removable denture from its basal seat. ${ }^{1}$ It is also called as the 'Occlusal Disharmony' or 'Occlusal Prematurity.' A lot more is discussed in the literature about the occlusal prematurities. The term 'proximal prematurity' does not exist in the GPT and little information is available about detecting and managing the same. However, the 'proximal contact' is defined in the GPT, as the area of a tooth that is in close association, connection, or touch with an adjacent tooth in the same arch. ${ }^{1}$ During the process of seating or fitting of any removable partial or fixed dental prosthesis including implant-fixed restorations, the first area to be observed before seating of the prosthesis in its final position is the 'proximal contact'. It is the

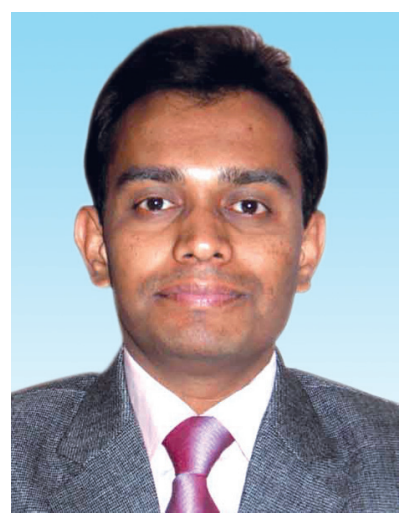
most critical area to manage during prosthesis fit. Proper proximal contact plays an important role in maintaining and stabilizing the dental arch. ${ }^{2}$

The weak or slightly opened proximal contact may cause food impaction, dental caries, periodontal disease, failure of occlusion and an undesirable drift of the teeth. On the other hand, too tight contact can damage periodontal ligament fibers of concern teeth and/or implant-bone interfaces or interfere with the physiological displacement of the teeth. ${ }^{3}$ Newell et al ${ }^{4}$ found that although food impaction is generally associated with open contacts, food impaction can also occur in interproximal sites when contacts are tight. The proximal contact strength (PCS) during tooth restoration is generally determined by the floss that passes through the contact point. ${ }^{3}$ Campagni ${ }^{5}$ described that the entry of floss with a snap indicates the proper proximal contact in clinical treatment. Normally the proximal contacts of indirect restorations are marked using articulating paper and adjusted with rotary instruments. Any nonspecific trimming of the prosthesis to eliminate the proximal prematurities may leave open or tight contacts which are nonmeasurable. Kim and Suh ${ }^{6}$ described the proximal contact adjustment and interproximal 'relief' method by using ultra-thin abrasive diamond dental strips (ContacEZ; ContacEZ,Vancouver, Wash) of various thicknesses. By restoring the proper interproximal contacts, complete seating of the indirect restoration can be achieved, and the occlusal interferences can be prevented. ${ }^{6}$

Detecting and adjusting the proximal prematurities in removable partial denture around the remaining natural teeth also is the difficult task. Careful parallel-block-out of the final cast before wax pattern fabrication and/or acrylization minimizes this difficulty. There is a need of development of concrete guidelines for identifying and eliminating the proximal prematurities in both removable and fixed dental prostheses.

\section{REFERENCES}

1. The academy of prosthodontics. The glossary of prosthodontic terms. 8th ed. J Prosthet Dent 2005;94(1):10-92.

2. Wheeler RC. An atlas of tooth form. 4th ed. Philadelphia, WB Saunders Co;1969:12.

3. Kim HS, Na HJ, Kim HJ, Kang DW, Oh SH. Evaluation of proximal contact strength by postural changes J Adv Prosthodont 2009;1(3):118-112.

4. Newell DH, John V, Kim SJ. A technique of occlusal adjustment for food impaction in the presence of tight proximal contacts. Oper Dent 2002;27(1):95-100.

5. Campagni WV. The final touch in the delivery of a fixed prosthesis. CDA J 1984;12:21-29.

6. Kim DS, Suh KW. A proximal contact adjustment and interproximal relief method. J Prosthet Dent 2007;97:244-245.

Pravinkumar G Patil Managing Editor International Journal of Prosthodontics and Restorative Dentistry

Division of Clinical Dentistry

School of Dentistry, International Medical University Kuala Lumpur, Malaysia 